\title{
Reduction of Reactor Weight by Using Electromagnetic Coupling
}

\author{
Takayuki NAKAMURA \\ Researcher,
}

Traction Control Laboratory, Vehicle Control Technology Division

\author{
Masamichi OGASA \\ Director, \\ Vehicle Control Technology Division
}

\begin{abstract}
In order to reduce joule loss due to ripple current in multiphase current reversible chopper, an attempt was made to use electromagnetic coupling for an air-core reactor. The relationships among the amplitude of ripple current, duty factor and electromagnetic-coupling coefficient was deduced. It turned out that a specific optimal coefficient of electromagnetic coupling reduces current ripple both on each phase and on total combined. The hypothesis was verified experimentally with newly manufactured reactors, which achieved the optimal electromagnetic coefficient. The weight of the new reactor is $44 \%$ lighter than conventional three-phase reactors.
\end{abstract}

Keywords: chopper, reactor, ripple current, duty factor, coupling coefficient

\section{Introduction}

Remarkable progress has been made in energy storage technology through high-performance energy storage devices opening the possibility to have train-onboard energy storage systems. However, energy storage devices on board trains must be accompanied by on-board DC-DC converter systems. For example, the "Hi-tram," developed by RTRI, has two three-phase current reversible choppers [1]. Onboard DC-DC converter systems increase vehicle weight. Ideally systems should both reduce harmonics (ripple current) and be physically lighter. A common approach to reducing overall ripple current in multiphase choppers is to increase the number of phases and shift-phase switching operation [2]. However, the multiphase chopper requires many essential components, such as semiconductors and smoothing reactors. Employing the smoothing reactors in particular adds to the chopper' $\mathrm{s}$ bulk and weight due to associated measures to avoid electromagnetic interference. The objective therefore is to have a system which is capable of both reducing ripple current and light weight.

Use of iron-core reactors is a common way to obtain necessary inductance. However when reactors are used on railway vehicles, high self-inductance should be also guaranteed with large current, therefore air-core reactors are generally preferred for railway vehicles. Ideally onboard air-core reactors should keep ripple current to a minimum and be compact and light weight.

Electromagnetic coupling is one means to achieve the double requirement of having a compact and light weight reactor while guaranteeing inductance. Use of electromagnetic coupling can reduce the heat produced by ripple current and the size of the accompanying cooling system, and thereby can achieve a more compact whole system. It is important to grasp quantitatively the relationship between the amplitude of ripple current and the electromagneticcoupling coefficient to reduce the ripple current.

The authors of this paper therefore deduced the re- lationships among the amplitude of ripple current, duty factor and electromagnetic-coupling coefficient. The ratio of the amplitude of ripple current was introduced to determine the loss and gain on each phase and on the total combined of multiphase chopper system in relation to that of the ripple current in single-phase chopper. The conditions needed to reduce the amplitude of ripple current is achieved both on each phase and on the total combined [3]. A reactor was then manufactured to attempt to achieve the optimal electromagnetic coefficient, and tested. The total loss of ripple current of manufactured reactors is less by $69 \%$ than that of conventional three-phase reactors, without electromagnetic coupling. The weight of the new reactor is $44 \%$ lighter than the conventional three-phase reactors.

\section{Theoretical value of the amplitude of ripple cur- rent}

\subsection{Deducing the theoretical value}

This paper focuses on the reactors in multiphase current reversible chopper (Fig. 1). Since the movement of the upper and lower arms in each leg are complementarily, current is continuous. The present paper regards the chopper as buck chopper. When the value of output DC current $i_{D C}$ is less than zero, the chopper operates as a boost chopper. There are two kinds of ripple current, each with a different value. One ripple current called "ph" originates from each phase in the chopper system while the other called "sum" originates from the total combined in the chopper system. When a multiphase chopper operates with shiftphase switching, a common consequence is a reduction on the total combined in multiphase chopper system. From the view point of reactor' $\mathrm{s}$ heat by joule loss, we also have to reduce the ripple current on each phase in multiphase chopper system. 


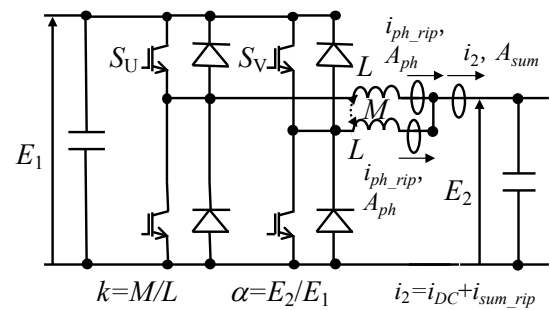

Fig. 1 Multiphase buck chopper (two phases)

Employing a multiphase chopper increases the number of phases and makes the control system more complex. Thus, the number of phase decreases from three to two. This paper clarifies how electromagnetic coupling influences the amplitude of ripple current in shift-phase switching. For this clarification, the present paper introduces the ratio of the amplitude of ripple current in each phase and in the total combined of multiphase chopper system to that of the ripple current in single-phase chopper. The ratio of less than one indicates that the electromagnetic coupling is effective in reducing the ripple current.

The slope of the phase current leads to the amplitude of the ripple current (Fig. 2). Using (1), it is possible to calculate the amplitude of the ripple current. The equation differs according to the state of the upper arm. $i_{\text {on }}$ is the phase current when the upper arm is on, $i_{\text {off }}$ is the phase current when the upper arm is off.

$$
\text { on: } i_{r i p}=\frac{T_{o n}}{2} \frac{d i_{o n}}{d t} \quad \text {, off: } i_{r i p}=\frac{T_{o f f}}{2} \frac{d i_{o f f}}{d t}
$$

Current consists of DC component $i_{D C}$ and ripple component $i_{r i p}$. Since the slope of $i_{D C}$ is zero, $i_{D C}$ and $i_{r i p}$ are independent of each other.

For single-phase buck chopper, (2) and (3) are the voltage equations, according to the state of the upper arm.

$$
\text { on: } L \frac{d i}{d t}=E_{1}-E_{2}
$$

off: $L \frac{d i}{d t}=-E_{2}$

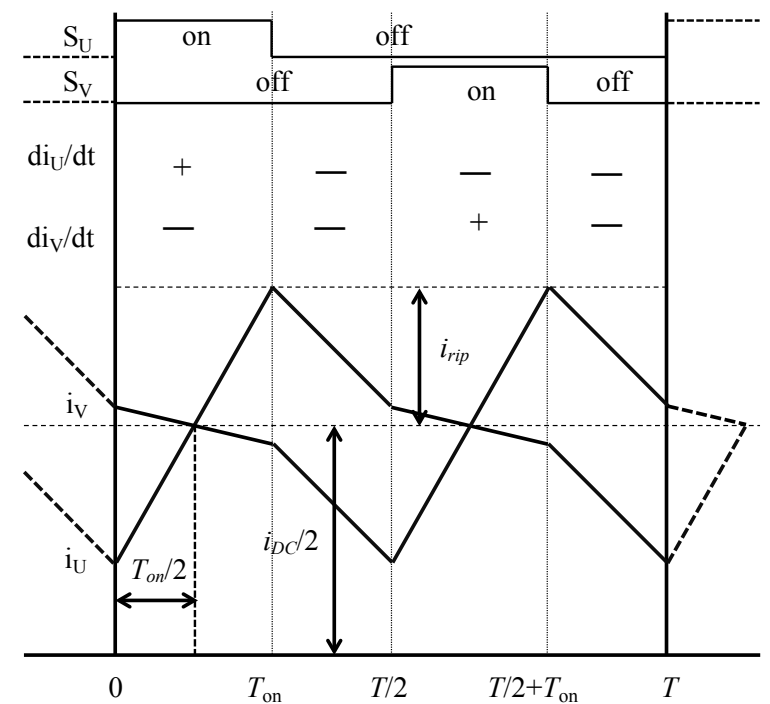

Fig. 2 Ripple current (two phases, shift-phase switching, $\alpha \leqq 0.5)$
The equations (1) to (3) lead to (4) to provide the amplitude of the ripple current on the single-phase chopper system.

$$
i_{\text {rip }}=\frac{E_{1} \alpha}{2 f L}(1-\alpha)
$$

$f$ is the frequency of the chopper, which for this paper is $700 \mathrm{~Hz} . \alpha$ indicates the duty factor. Since current is continuous, $\alpha$ is equal to $E_{2}$ divided by $E_{1}$.

For the two-phase buck chopper, (5) to (8) express the voltage equations on each phase, according to the state of the upper arm.

$$
\begin{array}{ll}
\mathrm{S}_{\mathrm{U}} \text { on: } & L \frac{d i_{U}}{d t}+M \frac{d i_{V}}{d t}=E_{1}-E_{2} \\
\mathrm{~S}_{\mathrm{U}} \text { off: } \quad & L \frac{d i_{U}}{d t}+M \frac{d i_{V}}{d t}=-E_{2} \\
\mathrm{~S}_{\mathrm{V}} \text { on: } & M \frac{d i_{U}}{d t}+L \frac{d i_{V}}{d t}=E_{1}-E_{2} \\
\mathrm{~S}_{\mathrm{V}} \text { off: } \quad M \frac{d i_{U}}{d t}+L \frac{d i_{V}}{d t}=-E_{2}
\end{array}
$$

When the two-phase current reversible chopper operates with shift-phase switching and the duty factor falls below 0.5 , (9) and (10) express the amplitude of the ripple current on each phase $i_{\text {ph_rip }}$, and on the total combined $i_{\text {sum rip }}$, respectively. The equations (5) to (8) lead to the slope of current, to obtain (9) and (10). When the duty factor exceeds 0.5 , each value is obtained by substituting $1-\alpha$ for $\alpha$.

$$
\begin{aligned}
& i_{\text {ph_rip }}=\frac{E_{1}}{2 f L} \frac{\alpha}{1+k}\left(\frac{1}{1-k}-\alpha\right) \\
& i_{\text {sum_rip }}=\frac{E_{1}}{2 f L} \frac{\alpha(1-2 \alpha)}{1+k}
\end{aligned}
$$

$k$ is the electromagnetic-coupling coefficient, which indicates the ratio of mutual-inductance to self-inductance. When $k$ exceeds zero, the reactors operate with cumulative coupling. When $k$ is less than zero, the reactors operate with differential coupling.

Dividing (9) and (10) by (4), produces the ratio of the amplitude of the ripple current on each phase and on the total combined of multiphase chopper system to that of the ripple current in single-phase chopper $A_{p h}$ (Fig. 3) and $A_{\text {sum }}$ (Fig. 4) respectively.

$$
A_{p h}=\frac{1}{(1+k)(1-\alpha)}\left(\frac{1}{1-k}-\alpha\right)
$$

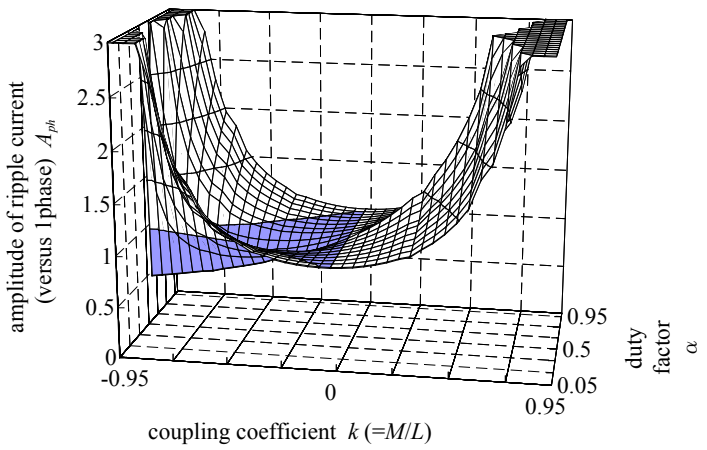

Fig. 3 Amplitude of ripple current on each phase chopper (two-phase chopper) 


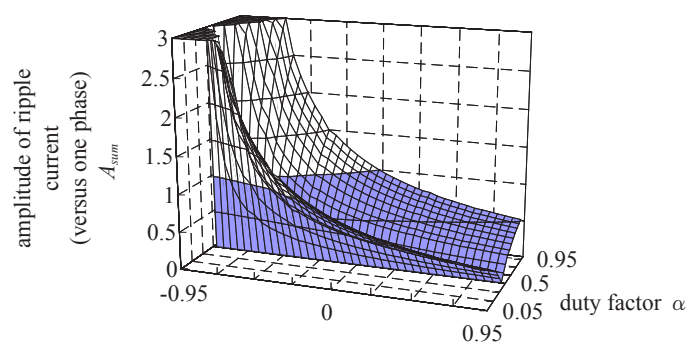

coupling coefficient $k(=M / L)$

Fig. 4 Amplitude of ripple current on total combined (two-phase chopper))

$$
A_{\text {sum }}=\frac{1}{1+k} \cdot \frac{1-2 \alpha}{1-\alpha}
$$

As shown in Fig. 3, it is known that a minimum value of $A_{p h}$ exists. The analytically obtained value is accurately determined by (13). $k_{\min }$ is the electromagnetic-coupling coefficient when $A_{p h}$ is at the minimum value. Equation (14) expresses $k_{\min }$

$$
\begin{aligned}
& A_{p h_{-} \min }=\frac{1}{1-\alpha}\left(\frac{\alpha}{1-\sqrt{1-2 \alpha}}\right)^{2} \\
& k_{\min }=1+\frac{\sqrt{1-2 \alpha}-1}{\alpha}
\end{aligned}
$$

For $k_{0}<k<0$, both $A_{p h}$ and $A_{\text {sum }}$ are less than one. $k_{0}$ is the electromagnetic-coupling coefficient corresponding to $A_{p h}=1$ and $A_{s u m}=1$. Equation (15) expresses $k_{0}$.

$$
k_{0}=-\frac{\alpha}{1-\alpha}
$$

To reduce the abovementioned ratio to less than one, the following conditions have to be satisfied when the twophase chopper operates with shift-phase switching. The efficient area is shown in Fig.5, in which the dashed lines indicate $k_{\min }$

-The absolute value of $k$ should be less than $|k|$.

-Reactors should operate with differential coupling.

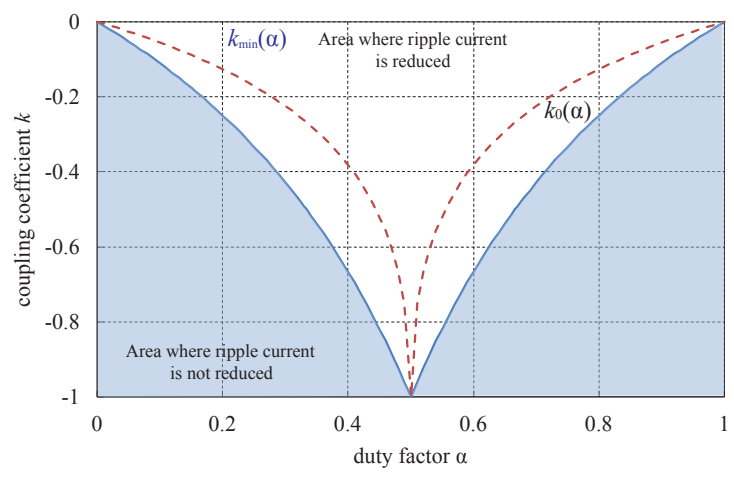

Fig. 5 Area where ripple current is reduced

\subsection{Selection of electromagnetic coupling coeffi- cients}

This paper then considered application of the electromagnetic coupling of reactor to the design of an energy storage system on a train. The energy storage system onboard train is necessary to the chopper system for charging and discharging. For example, actual vehicles with onboard energy storage systems use a chopper to convert DC 1,500 V/600 V into DC $750 \mathrm{~V}$ [1]. This paper estimates the effect of electromagnetic coupling when $E_{1}=1,500[\mathrm{~V}]$, $\alpha=0.4, L=4.3[\mathrm{mH}]$, and $f=700[\mathrm{~Hz}]$. Since trolley voltage changes from $1,200 \mathrm{~V}$ to $1,800 \mathrm{~V}$, the duty factor range $\alpha$ is $0.42 \leq \alpha \leq 0.62$. The electromagnetic-coupling coefficient should therefore be selected to ensure, it corresponds to $k_{0}$ $<k<0$ in the duty factor range. Figure 5 shows that, the optimal area of electromagnetic-coupling coefficient lies between $-0.61<k<0$. When $\alpha=0.45, k_{0}, k_{\min }$, and $A_{p h \text { min }}$ become $-0.81,-0.52$, and 0.79 , respectively. Therefore, when an electromagnetic-coupling air-core reactor is used to the onboard DC-DC converter system onboard train, the optimal electromagnetic-coupling coefficient is around -0.5 .

\section{Manufacture of reactors}

Previous chapters have demonstrated that it is possible to reduce ripple current, when the two-phase chopper operates with optimal electromagnetic coupling. Such a decrease can only be achieved if the reactors provide an optimum electromagnetic-coupling coefficient.

\subsection{Existing reactors}

Existing reactors, shown in Fig. 6, are used on the chopper systems of electric vehicle developed by RTRI [1]. It has three phases and choppers operate with equal-phase switching. There are no electromagnetic couplings among reactors on the chopper systems. Table 1 gives the specifications of these reactors.

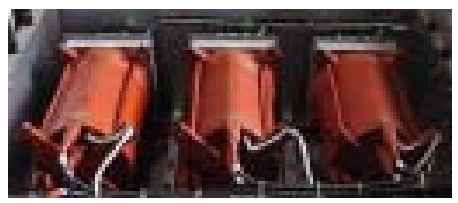

Fig. 6 Onboard reactors

\subsection{The new reactors}

To achieve optimal electromagnetic-coupling coefficient a new reactor structure with two distinctive features was developed. Firstly the new reactors are wound separately (Fig. 7, $x$ turns), then the remaining area are wound together (Fig. 7, $y$ turns). Where the ratio of $x$ to $y$ changes, the electromagnetic-coupling coefficient changes (Fig. 7).

\subsection{Manufacture of electromagnetic-coupling reac- tors}

By adopting the abovementioned structure whereby, such that the ratio of $x$ to $y$ is one to one, the electromagnetic-coupling coefficient nears 0.5 . An example of a reactor using this method was then built (Fig. 8).

Table 1 shows the specifications of the manufactured reactor. These values are used in the calculations below. Compared with the existing two-phase reactors, the mass was cut by $30 \mathrm{~kg}$, and by $44 \mathrm{~kg}$ compared with the existing three-phase reactors. 


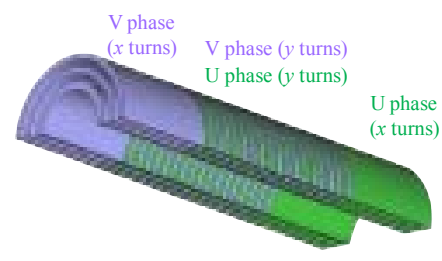

(a) $x: y=1: 1 \quad(k \approx 0.5)$

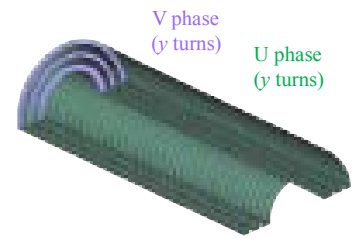

(b) $x: y=0: 1 \quad(k \approx 1.0)$

Fig. 7 The structure of electromagnetic coupling reactor
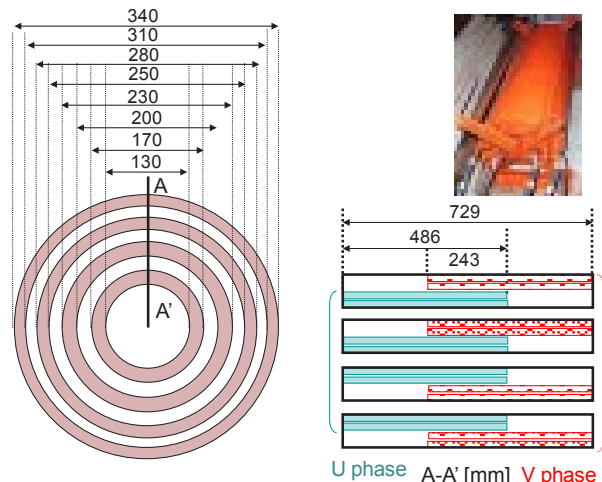

Fig. 8 The manufactured reactors

Table 1 Reactor specifications

\begin{tabular}{|c|c|c|}
\hline Reactor & $\begin{array}{c}k=0 \\
\text { (on LH02) }\end{array}$ & $\begin{array}{c}k=0.5 \\
\text { (Manufactured) }\end{array}$ \\
\hline Self-inductance & $4.3 \mathrm{mH}$ & $3.38 \mathrm{mH}$ \\
\hline Coupling coefficient & 0 & 0.60 \\
\hline Turns & 240 & 216 \\
\hline Mass (Total) & $\begin{array}{c}90 \mathrm{~kg} \\
(1 \mathrm{phase}) \times 3\end{array}$ & $150 \mathrm{~kg}$ \\
\hline $\begin{array}{c}\text { Mass (versus the } \\
\text { conventional two phase) }\end{array}$ & - & $-17 \%$ \\
\hline $\begin{array}{c}\text { Mass (versus the } \\
\text { conventional three phase })\end{array}$ & - & $-44 \%$ \\
\hline
\end{tabular}

\section{Test results of ripple current}

The new reactors were tested for the chopper systems, used in electric railway vehicles. Agreement of measured values with the theoretical values would confirm the theory explained in chapter 2 for reducing ripple current.

\subsection{Existing reactors}

Figure 9 shows the ripple current for output DC current $i_{D C}$ of zero. Figure 9 shows an input voltage $E_{1}$ of 1,675 $\mathrm{V}$, with $\alpha$ duty factor a of 0.435 . The measured values shown in Fig. 9 are $69.7 \mathrm{~A}$ (each phase) and 217.5 A (total combined).

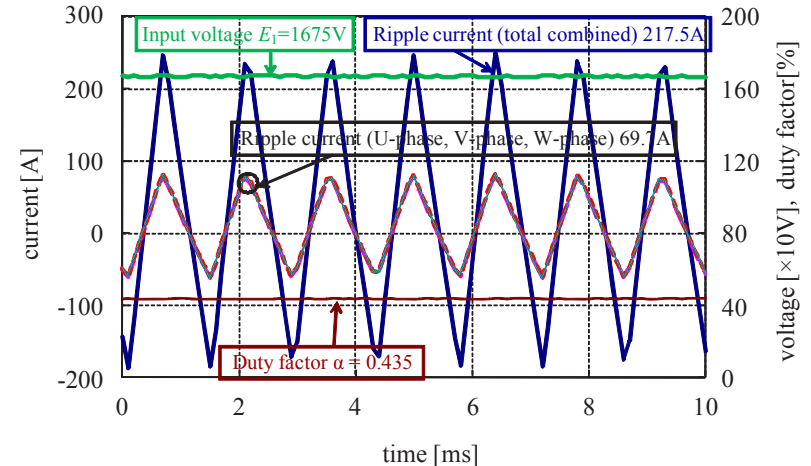

Fig. 9 Result of ripple current (three phases, existing reactors)

\subsection{New reactors}

As demonstrated in the chapter 2 , when using the new reactors in a two-phase chopper system, the best performance is achieved with shift-phase switching and differential coupling.

Figure 10 shows the results of ripple current when the output DC current $i_{D C}$ is zero. Table 2 shows the amplitude of the ripple current for output DC currents $i_{D C}$ of 0,120 and 360. As shown in Fig. 10, input voltage $E_{1}$ is $1,657 \mathrm{~V}$, and the duty factor $\alpha$ is 0.38 . The theoretical values of the ripple current on each phase and on the total combined are $82.0 \mathrm{~A}$ and $81.6 \mathrm{~A}$, respectively. The measured values shown in Fig. 10 are $83.4 \mathrm{~A}$ (U phase), $82.2 \mathrm{~A}$ (V phase), and $72.5 \mathrm{~A}$ (the total combined). The measured values agree with the theoretical values. Table 2 shows that the amplitude of the ripple current is independent of the output DC current.

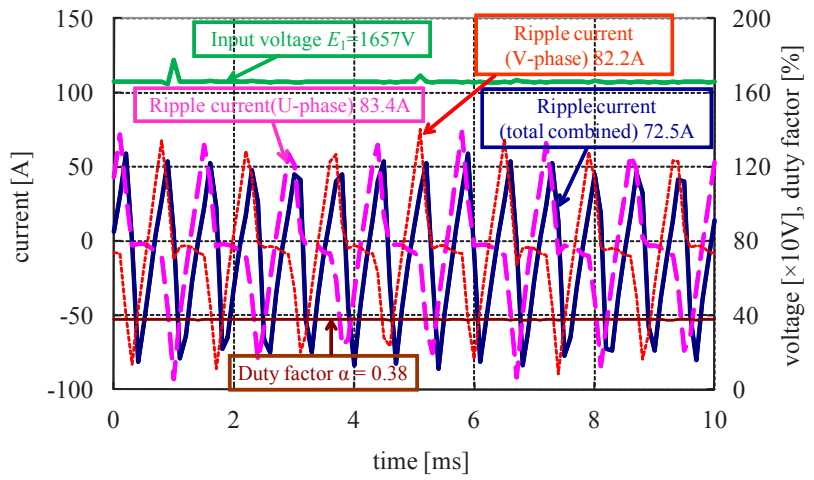

Fig. 10 Result of ripple current $(k=0.5$, differential coupling, shift-phase switching)

Table 2 Result of ripple current $(k=0.5$, differential coupling, shift-phase switching)

\begin{tabular}{|c|c|c|}
\hline \multicolumn{2}{|c|}{$i_{D C}$} & Amplitude of Ripple Current \\
\hline \multirow{2}{*}{$i_{D C}=0$} & $i_{\text {ph__ip }}[\mathrm{A}]$ & $\mathrm{U}: 83.4, \mathrm{~V}: 82.2(82.0)$ \\
\cline { 2 - 3 } & $i_{\text {sum_rip }}[\mathrm{A}]$ & $72.5(81.6)$ \\
\hline \multirow{2}{*}{$i_{D C}=120$} & $i_{\text {ph_rip }}[\mathrm{A}]$ & $\mathrm{U}: 78.1, \mathrm{~V}: 75.6(77.1)$ \\
\cline { 2 - 3 } & $i_{\text {sum_rip }}[\mathrm{A}]$ & $65.0(65.3)$ \\
\hline \multirow{2}{*}{$i_{D C}=360$} & $i_{\text {ph__ip }}[\mathrm{A}]$ & $\mathrm{U}: 74.4, \mathrm{~V}: 73.8(77.1)$ \\
\cline { 2 - 3 } & $i_{\text {sum_rip }}[\mathrm{A}]$ & $63.8(55.3)$ \\
\hline
\end{tabular}

$※$ inside ( ): theoretical values 
This result confirms that when the new reactors $(k=$ $0.5)$ are used in a two-phase chopper, the best performance is achieved with shift-phase switching and differential coupling. When the duty factor $\alpha$ is $0.4, k_{\min }$ becomes -0.38 by (10). If the electromagnetic-coupling coefficient $k$ is 0.4 and the chopper operates with shift-phase switching and differential coupling, then the ripple current could be further reduced.

\section{Test results of loss}

Previous sections, in this paper have dealt with the effect of electromagnetic coupling on the amplitude of the ripple current. When electromagnetic coupling is applied to the multiphase chopper, it is necessary to also consider joule loss relating to not only to the ripple current but also to the DC current. This section therefore focuses on joule loss. There are two kinds of loss. One originates from the ripple current on each phase in the reactor of multiphase chopper system. The other is due to the ripple current on the total combined in the multiphase chopper system at loads etc. The square of current leads to joule loss, which consists of the square of the DC current, the square of the ripple current and the product of the DC current and ripple current. Since the ripple current is expressed by a periodic function, the third component becomes zero. When $r, R, n$ are the resistance of reactor, the resistance of load, and number of phase of multiphase chopper respectively, (16) indicates total losses. Since the waveform of the ripple current is a triangular wave, it is obtained by dividing the RMS value $i_{\text {rip }}$ by $\sqrt{3}$. In (16), the former term indicates the loss of the reactors, and the latter term indicates the loss in loads.

$$
W=r\left\{\left(\frac{i_{D C}}{n}\right)^{2}+\left(\frac{i_{\text {ph_rip }}}{\sqrt{3}}\right)^{2}\right\} \times n+R\left\{i_{D C}^{2}+\left(\frac{i_{\text {sum_rip }}}{\sqrt{3}}\right)^{2}\right\}
$$

When calculating reactor losses, the only factor is the amplitude of the ripple current on each phase in the multiphase chopper system. When calculating load losses, the only factor is the amplitude of ripple current on the total combined in the multiphase chopper system. However, there are rare occasions on which both the amplitude of ripple current on each phase and the amplitude of ripple current on the total combined can be ignored. This paper therefore considers the condition where $r$ is equal to $R$. Accordingly, the effects of both the amplitude of the ripple currents appear on a near equal level. The estimation of loss is equivalent to the estimation of the square of the current. Under this condition, the indication of loss is obtained by dividing (16) divided by $r$.

$$
\text { Loss }=\frac{n+1}{n} i_{D C}{ }^{2}+\frac{n}{3} i_{\text {ph_rip }}{ }^{2}+\frac{1}{3} i_{\text {sum_rip }}{ }^{2}
$$

When a three-phase chopper employs conventional reactors, Loss becomes $20,627\left[\mathrm{~A}^{2}\right]$ for $i_{D C}=0$ by (17). When a two-phase chopper uses new reactors and operates with differential coupling, Loss becomes 6,323 [ $\left.\mathrm{A}^{2}\right]$ by (17). The ratio of the indication to the existing becomes 0.31 (Table 3 ). These results mean that if the chopper operates in the abovementioned means, ripple current is reduced by nearly
$70 \%$. If $i_{D C}$ becomes infinite, these ratios converge to 1.125 $(=9 / 8)$. There is a break-even point around $i_{D C}=270 \mathrm{~A}$. The electromagnetic coupling reactors is used on vehicles which run with a hybrid power source from contact wires and/or onboard batteries. The vehicle developed by RTRI needs about $270 \mathrm{~A}$ (RMS) to run $300 \mathrm{~m}$ up to $40 \mathrm{~km} / \mathrm{h}$. For this current, although the number of phase decreases, the loss of the two-phase systems is the same as that of the basic system.

\begin{tabular}{|c|c|c|c|c|}
\hline \multicolumn{2}{|c|}{ Reactor } & $k=0$ & \multicolumn{2}{|c|}{$k=0.5$} \\
\hline \multicolumn{2}{|c|}{$\begin{array}{l}\text { Switching } \\
\text { (Phase) }\end{array}$} & $\begin{array}{c}\text { Equal } \\
\left(0^{\circ}\right)\end{array}$ & \multicolumn{2}{|c|}{ Shift $\left(180^{\circ}\right)$} \\
\hline \multicolumn{2}{|c|}{ Coupling } & - & \multicolumn{2}{|c|}{ Differential } \\
\hline \multicolumn{2}{|c|}{$\operatorname{Loss}\left[\mathrm{A}^{2}\right]$} & $\begin{array}{c}\operatorname{Loss}_{3_{-} E} \\
{\left[\mathrm{~A}^{2}\right]}\end{array}$ & $\begin{array}{c}\operatorname{Loss}_{2 \_D} \\
{\left[\mathrm{~A}^{2}\right]}\end{array}$ & $\begin{array}{l}\text { Ratio to } \\
\operatorname{Loss}_{3 \_} E\end{array}$ \\
\hline \multirow{4}{*}{$i_{D C}[\mathrm{~A}]$} & 0 & 20,627 & 6,323 & 0.307 \\
\hline & 120 & 39,827 & 26,951 & 0.676 \\
\hline & 360 & 193,427 & 199,417 & 1.031 \\
\hline & $\infty$ & $\infty$ & $\infty$ & 1.125 \\
\hline
\end{tabular}

Table 3 The index and ratio of loss

\section{Conclusion}

This paper deals with electromagnetic coupling reactors in two-phase current reversible chopper system. This paper focuses in particular on identifying the optimal electromagnetic-coupling coefficient. This paper also estimates the loss and gain when applying these reactors and switching to railway vehicles, equipped with onboard energy storage systems.

- The relationships is deduced among the amplitude of ripple current, duty factor and electromagnetic coupling coefficient. To determine the loss and gain, a ratio is introduced of the amplitude of the ripple current in each phase and in total combined of multiphase chopper system to that of the ripple current in single-phase chopper.

- From the deduction, it is determined to achieve considerable electromagnetic coupling efficiency, the twophase chopper should operate with differential coupling and the absolute value of the electromagneticcoupling coefficient should be less than $\left|k_{0}\right|$, which corresponds to $A_{p h}=1$ and $A_{\text {sum }}=1$.

- A new structure was developed which provides a large and optimal electromagnetic-coupling coefficient. A new reactors was built on this new design with an electromagnetic-coupling coefficient of 0.6 . The new reactor was $44 \%$ lighter than existing three phase reactors.

- The loss from ripple current was reduced to $31 \%$, when the chopper operated with shift-phase switching and differential coupling, and the new reactor. When the loss relating to DC current is included, the abovementioned losses smaller than in a existing three-phase system. If $i_{D C}$ is less than the value of electric railway vehicle running $300 \mathrm{~m}$ with $40 \mathrm{~km} / \mathrm{h}$ (270 A).

We ensure the reduction in ripple current and loss, by using electromagnetic coupling. The abovementioned methods will reduce the mass and volume of DC-DC choppers. 


\section{Acknowledgment}

This study was promoted under a contract with the NEDO (New Energy and Industrial Technology Development Organization).

\section{References}

[1] M. Ogasa : “Application of Energy Storage Technolo- gies for Electric Railway Vehicles - Examples with Hybrid Electric Railway Vehicles," IEEJ Trans. Electrical and Electronic Engineering, Vol. 5, No.3, pp. $304-311$, 2010.

[2] IEEJ, Power Electronics Circuit, 2000.

[3] T. Nakamura, Y. Taguchi, M. Ogasa : "Reduction in Ripple Current and Self-inductance by Using Electromagnetic Coupling of Reactor," IEEJ Trans., Vol.132D, No.7, pp. 736 - 746, 2012. 